\title{
Consensus Statement on Natural Orifice Transluminal Endoscopic Surgery and Single-Incision Laparoscopic Surgery: Heralding a New Era in Urology?
}

\author{
Matthew T. Gettman ${ }^{a, *}$, Geoffrey Box ${ }^{b}$, Timothy Averch ${ }^{c}$, Jeffrey A. Cadeddu ${ }^{d}$, \\ Edward Cherullo ${ }^{e}$, Ralph V. Clayman ${ }^{b}$, Mihr Desai ${ }^{f}$, Igor Frank ${ }^{a}$, Indebir Gill ${ }^{f}$, \\ Mantu Gupta ${ }^{g}$, Georges-Pascal Haber ${ }^{f}$, Mitchell Humphreys ${ }^{a}$, Jihad Kaouk ${ }^{f}$, \\ Jaime Landman ${ }^{g}$, Estevao Lima ${ }^{h}$, Lee Ponsky ${ }^{e}$ \\ ${ }^{a}$ Mayo Clinic, Department of Urology, Rochester, MN, United States \\ ${ }^{\mathrm{b}}$ University of California Irvine, CA, United States \\ ${ }^{\mathrm{c}}$ University of Pittsburgh Medical Center, PA, United States \\ ${ }^{\mathrm{d}}$ University of Texas Southwestern Medical Center, Dallas, TX, United States \\ ${ }^{\mathrm{e}}$ Case Western Reserve University, Cleveland, $\mathrm{OH}$, United States \\ ${ }^{\mathrm{f}}$ Cleveland Clinic, Cleveland, $\mathrm{OH}$, United States \\ ${ }^{g}$ Columbia University Medical Center, New York, NY, United States \\ ${ }^{\mathrm{h}}$ University of Minho, School of Health Science, Braga, Portugal
}

For decades, urologists have implemented technologies that provide effective treatment while limiting morbidity. In many instances, this has been achieved by operating via natural body openings (eg, cystoscopy, transurethral resection, and ureteroscopy) [1,2]. Urologists have also pioneered novel techniques to address clinical situations where access through natural body openings was impossible, such as percutaneous stone surgery, laparoscopy, and robotics [3-5].

In the last $5 \mathrm{yr}$, exponential development of therapeutic endoscopy has been realized. There is interest now in developing surgical procedures that enter the peritoneum or retroperitoneum through hollow viscera that can be assessed via natural body openings precluding skin incisions [6,7]. The new approaches, coined natural orifice transluminal endoscopic surgery (NOTES), aim to further limit treatment morbidity, but studies addressing these approaches have yet to be initiated because the clinical techniques for these approaches are being developed [6]. NOTES can be performed as a pure procedure involving one portal of entry or as a combined procedure involving use of multiple body openings. NOTES can also be performed as hybrid procedures in conjunction with conventional transabdominal ports. Closely related to NOTES is development of procedures performed through a solitary small transabdominal incision. These procedures have been referred to as single-port access, single- port laparoscopy, or single-incision laparoscopy; consensus on the most appropriate name for the approach has not been achieved. More importantly, the "it's new, but is it better" question again has yet to be addressed for these procedures, because they remain in their nascent forms.

Urology is favorably positioned for development of NOTES technology and introduction of new

* Corresponding author. Mayo Clinic, Department of Urology, 200 First Street SW, Rochester, MN 55905, United States.

Tel. +1 507281 9795; Fax: +1 5072844951 .

E-mail address: gettman.matthew@mayo.edu (M.T. Gettman).

0302-2838/\$ - see back matter (C) 2008 European Association of Urology. Published by Elsevier B.V. All rights reserved. doi:10.1016/j.eururo.2008.02.006 
surgical approaches given our training in open surgical, endoscopic, and image-guided techniques. Urologists' long history and experience with these minimally invasive technologies give us an enormous advantage as we further develop and evaluate NOTES and single-incision procedures. In contrast to urology, other specialties are experiencing blurring of traditional procedural boundaries as new approaches are introduced. Specialties preferentially interested in diagnostic procedures are now interested in therapeutic interventions. On the other hand, specialties previously interested in predominantly open therapeutic interventions are now interested in diagnostic techniques or minimally invasive therapies.

Experimentally, urologists have been at the forefront of NOTES and procedures performed through solitary small abdominal incisions. The clinical event prompting experimental evaluation of urologic NOTES was the description of vaginal extraction of an intact surgical specimen following laparoscopic radical nephrectomy [8,9]. In 2002, Gettman et al described the first experimental application of natural orifice surgery when transvaginal nephrectomy was successfully performed in the porcine model [10]. Indeed, this work predated the acronym NOTES as well as the first recognized NOTES report by Kalloo et al in the gastroenterology literature, which was not reported until 2004 [11]. Likewise, the bladder was successfully used by Lima et al as a NOTES portal in experimental models [12]. Combined NOTES approaches involving transgastric and transvesical access have also been reported experimentally by the same group for nephrectomy and cholecystectomy [13,14] as well as thoracoscopy [15]. In addition, another combined NOTES nephrectomy technique was recently described by Ponsky et al using combined transgastric and transvaginal access [16]. NOTES operative platforms have also been engendered and evaluated by urologists. The magnetic anchoring and guidance system (MAGS) was introduced for active camera and instrument control. With MAGS, transvaginal nephrectomies and cholecystectomies have been performed experimentally $[17,18]$ A TransPort multilumen operating platform (USGI Medical, San Clemente, CA) has also been successfully used by Clayman et al for hybrid transvaginal NOTES nephrectomy in the porcine model [19]. In addition, transvaginal hybrid NOTES nephrectomy has been performed experimentally with the da VinciS robot (Intuitive Surgical, Sunnyvale, CA) [20].

The new NOTES surgical techniques are also gaining momentum clinically. Branco et al described hybrid NOTES transvaginal nephrectomy involving vaginal placement of an endoscope and two 5-mm abdominal trocars to remove a nonfunctioning right kidney. Operative time was $170 \mathrm{~min}$ and no complications occurred [21]. Using novel instrumentation, single-incision laparoscopic surgery has emerged as another important clinical development. The pioneering work in urology was first described at the University of Texas Southwestern Medical Center and soon after at Cleveland Clinic [22-24]. The first single-incision transumbilical three-port nephrectomy was performed at the University of Texas Southwestern Medical Center. The first single-port transumbilical nephrectomy was performed at Cleveland Clinic. To date, researchers have routinely used this approach clinically for pyeloplasty and nephrectomy, and also prostatectomy and ileal ureter substitution, and donor nephrectomy (pers. comm., J. Kauok, Cleveland, OH, USA). At Case Western Reserve University, urologists have performed single-access site laparoscopic radical nephrectomy, whereby all instrumentation was placed through one 7-cm incision [25]. In 2007, Gettman and Blute also reported the initial clinical NOTES experience in urology with transvesical peritoneoscopy used to evaluate suprapubic tube placement in conjunction with robotic prostatectomy [7].

The opportunity for the new NOTES approaches is enormous, yet the technology is still very much in evolution and methods themselves must be carefully implemented and then scientifically evaluated. Active participation by urologists is mandatory in this regard. To discuss the emerging roles in urology for NOTES and procedures performed through solitary small abdominal incisions, 16 members of the Endourological Society met in Cancun, Mexico on November 1, 2007 during the 25th World Congress of Endourology. This was simply an ad hoc group of interested individuals not necessarily including all thought leaders in the field. In fact, the group welcomes additional participants at future meetings. Topics relevant to development of urologic NOTES were discussed and a working group named the Urology Working Group on NOTES was formed. Stated working group goals are to:

- Increase awareness of NOTES in urology

- Provide an outlet to share discoveries related to urologic NOTES

- Guide scientific evaluation and implementation of urologic NOTES

- Facilitate learning opportunities with urologic NOTES

- Define nomenclature of urologic NOTES 
The vision of the working group is to safely and systematically implement NOTES in urology. We are well aware of the often cited statement: "everything good was once new, but everything new is not necessarily good." The group remains firmly committed to evaluating the new approach once it has gone through formative technological and learning curve stages.

The working group will formally meet during major urologic meetings sponsored by the Endourological Society, American Urological Association, and the European Association of Urology. During these meeting, both didactic and research forums will be held. Didactic sessions will be open to all conference participants; however, formal inclusion in the working group will mandate that one has published or presented experimental or clinical work related to NOTES. Members are expected to actively participate in the research forum by providing brief summaries of their most recent NOTES activities. The first meeting of this nature has been scheduled through the auspices of the Endourological Society and will take place at the American Urological Association meeting on Saturday May 17, 2008.

At the initial meeting it was critical to the working group that urologic NOTES procedures must be performed by urologists, regardless of the portals of entry, because urologists are experts of diseases involving the urinary tract. Teams of surgeons are recommended for combined or hybrid urologic procedures involving non-urologic portals of entry, but the urologist should be the primary surgeon; however, if a transgastric portal was used, then the gastroenterologist would work with the urologist to open and close the portal. Similarly, for combined, pure, or hybrid NOTES procedures involving bladder access portals but non-urologic indications, urologists should also be the primary surgeon for access and closure of the bladder.

At the initial working group meeting, all participants agreed that pure NOTES (ie, transgastric, transcolonic, transvaginal, or transvesical) should be further developed as a research topic in urology before widespread clinical implementation. The rationale was simply that more barriers exist with pure NOTES than the other single small incision techniques. In addition, any NOTES clinical research at this time should be done under approval of an institutional review board. The group identifies lack of purpose-built endoscopes, instrumentation, and training as the most significant limitations presently for pure NOTES implementation; however, the group is actively addressing these concerns and developing solutions to these problems.

Based on the current state of the art, the working group concluded that procedures performed through solitary small abdominal incisions are immediately available for clinical implementation. The group recommends that outcomes be reported as part of a multi-institutional clinical study. Since the vision of the working group is that new technologies should be safely and systematically implemented, outcomes must be carefully tracked. To this end, a clinical registry and database are in the process of being established as a requisite for NOTES working group members.

As a premise it is also thought that the new urologic NOTES techniques including the single small abdominal incision techniques must match or exceed the efficacy and safety of other accepted minimally invasive techniques for the emerging approaches to be justified. The realist in the mix will rightfully question the value of these new approaches; each must prove itself to be equal or better than current laparoscopic approaches in the realms of efficiency, effectiveness, economy, and equanimity (ie, patient convalescence, morbidity, pain, and cosmesis). It is conceivable that such may not be the case and that NOTES and single small abdominal incision techniques may prove to be a blip rather than a staple in surgical evolution. The working group sees it as their responsibility to accurately report and assess these techniques as applied to each procedure such that what is of value can be identified and taught and what is of no proven benefit can be discarded.

Enthusiasm for the single small abdominal incision techniques in urology is high given the similarities to traditional laparoscopic surgery. For instance, urologists are already familiar with access and closure methodologies at the umbilicus as well as management of complications related to conventional laparoscopy and robotics. In addition, urologists are already trained in surgical techniques and surgical principles required for the new approach. However, whether one port proves to be preferable to four or five smaller ports remains to be proven.

In summary, NOTES and single small abdominal incision techniques are gaining momentum and may represent the next frontier in minimally invasive surgery. Although many unanswered questions persist, it is also critically important that urologists maintain their pioneering spirit and evaluate these new approaches carefully and scientifically. Based predominantly on experimental results, pure NOTES should be actively pursued as 
a research topic. The single small abdominal incision techniques are immediately available for careful clinical implementation and as such require immediate inclusion in a clinical registry to determine their proper role in minimally invasive urologic surgery, lest they be promoted before they are tested and thus fall into the trap of other highly touted but poorly tested urologic procedures that subsequently took years to "unmask" and discard, much to the public's detriment. Indeed, a transition from standard laparoscopy to NOTEStype approaches is predicted only if this transition is supported by patient safety and clinical benefit. At the very least, evaluation of new technologies targeted for NOTES may well advance other existing urologic technologies such as percutaneous renal surgery, ureteroscopy, laparoscopy, or robotics. Furthermore, the working group adamantly supports the concept that urologists are uniquely situated to best evaluate NOTES approaches given the breadth of urologic training in open, endoscopic (cystoscopic, ureteroscopic, laparoscopic, percutaneous, and robotic) and image-guided (ultrasound and fluoroscopic) surgery.

\section{Conflicts of interest}

The authors have nothing to disclose.

\section{References}

[1] Rassweiler J, Teber D, Kuntz R, Hofmann R. Complications of transurethral resection of the prostate (TURP)-incidence, management, and prevention. Eur Urol 2006;50: 969-80.

[2] Geavlete P, Georgescu D, Mirciulescu V, Niță G. Ureteroscopic laser approach in recurrent ureteropelvic junction stenosis. Eur Urol 2007;51:1542-8.

[3] Michel MS, Trojan L, Rassweiller JJ. Complications in percutaneous nephrolithotomy. Eur Urol 2007;51:899-906.

[4] Clayman RV, Kavoussi LR, Soper NJ, et al. Laparoscopic nephrectomy: initial case report. J Urol 1991;146:278-82.

[5] Menon M, Shrivastava A, Kaul S, et al. Vattikuti Institute prostatectomy: contemporary technique and analysis of results. Eur Urol 2007;51:648-58.

[6] Rattner D, Kalloo A, ASGE/SAGES Working Group. ASGE/ SAGES working group on natural orifice transluminal endoscopic surgery. Surg Endosc 2006;20:329-33.

[7] Gettman MT, Blute ML. Transvesical peritoneoscopy: initial clinical evaluation of the bladder as a portal for natural orifice transluminal endoscopic surgery. Mayo Clinic Proc 2007;82:843-5.
[8] Gill IS, Cherullo EE, Meraney AM, et al. Vaginal extraction of the intact specimen following laparoscopic radical nephrectomy. J Urol 2002;167:238-41.

[9] Breda G, Silvestre P, Giunta A, et al. Laparoscopic nephrectomy with vaginal delivery of the intact kidney. Eur Urol 1993;24:116-7.

[10] Gettman MT, Lotan Y, Napper CA, Cadeddu JA. Transvaginal laparoscopic nephrectomy: development and feasibility in the porcine model. Urology 2002;59:446-50.

[11] Kalloo AN, Singh VK, Jagannath SB, et al. Flexible transgastric peritoneoscopy: a novel approach to diagnostic and therapeutic interventions in the peritoneal cavity. Gastrointest Endosc 2004;60:114-7.

[12] Lima E, Rolanda C, Pego J, et al. Transvesical endoscopic peritoneoscopy: a novel $5 \mathrm{~mm}$ port for intra-abdominal scarless surgery. J Urol 2006;176:802-5.

[13] Rolanda C, Lima E, Pego J, et al. Third-generation cholecystectomy by natural orifices: transgastric and transvesical combined approach. Gastrointest Endosc 2007;65:111-4.

[14] Lima E, Rolanda C, Pego J, et al. Third-generation nephrectomy by natural orifice transluminal endoscopic surgery. J Urol 2007;178:2648-54.

[15] Lima E, Henriques-Coelho T, Rolanda C, et al. Transvesical thoracoscopy: a natural orifice translumenal endoscopic approach for thoracic surgery. Surg Endosc 2007;21: 854-8.

[16] Ponsky L, Isariyawongse J, McGee M, et al. Pure natural orifice transluminal endoscopic surgery (NOTES) nephrectomy using standard laparoscopic instruments in the porcine model. J Endourol 2007;21:A91.

[17] Zeltser IS, Bergs R, Fernandez R, et al. Single trocar laparoscopic nephrectomy using magnetic anchoring and guidance system in the porcine model. J Urol 2007;178: 288-91.

[18] Scott DJ, Tang SJ, Fernandez R, et al. Completely transvaginal NOTES cholecystectomy using magnetically anchored instruments. Surg Endosc 2007;21:2308-16.

[19] Clayman RV, Box GN, Abraham JB, et al. Rapid communication: transvaginal single-port NOTES nephrectomy: initial laboratory experience. J Endourol 2007;21:640-4.

[20] Box GN, Lee HJ, Santo RJS, et al. Robot-assisted NOTES nephrectomy: initial report. J Endourol. In press.

[21] Branco AW, Branco Filho AJ, Kondo W, et al. Hybrid transvaginal nephrectomy. Eur Urol 2008;53:1290-4.

[22] Raman JD, Bensalah K, Bagrodia A, et al. Laboratory and clinical development of single keyhole umbilical nephrectomy. Urology 2007;70:1039-42.

[23] Desai MM, Rao PP, Aron M, et al. Scarless single port transumbilical nephrectomy and pyeloplasty: first clinical report. BJU Int 2008;101:83-8.

[24] Kaouk JH, Haber G-P, Goel R, et al. Single-port laparoscopic surgery in urology: initial experience. Urology 2007;71:3-6.

[25] Ponsky L, Cherullo E, Sawyer M, et al. Single access site laparoscopic radical nephrectomy: initial clinical experience. J Endourol. In press. 